AIAA

CONTROL OF SHOCK LOADING FROM A JET IN THE PRESENCE OF A FLEXIBLE STRUCTURE

\author{
Lucio Maestrello \\ NASA Langley Research Center \\ Hampton, Virginia
}

AIAA Paper No. 99-1975

Presented at the

5th AIAA/CEAS Aeroacoustic Conference

Bellevue, Washington

May 10-12, 1999 
ALAA-99-1975

\title{
CONTROL OF SHOCK LOADING FROM A JET IN THE PRESENCE OF A FLEXIBLE STRUCTURE
}

\author{
Lucio Maestrello* \\ NASA Langley Research Center, \\ Hampton, VA 23681-2199
}

\begin{abstract}
The control of shock noise or screech from a jet near a flexible structure is discussed. The pressure from the supersonic jet consists of a shock with spiral and flapping nonaxisymmetric modes superimposed on broadband response. This shock induces a nonlinearnonstationary loading problem associated with acoustic wave generation and propagation coupled with structural vibration. Control of the shock is achieved by placing a ring at the nozzle lip oscillating at the shock fundamental frequency. The ring prevents the shock characteristics originating in the column of the shear layer from sustaining connection with the out-of-phase surface vibration. Shock-free flow is maintained over a large pressure ratio. The peak power pressure level is reduced by $40 \mathrm{~dB}$.
\end{abstract}

\section{Background}

A shock-free jet can be achieved by activating the nozzle lip in a feedback loop which interferes with the shock formation. The idea is used to suppress shock loading and reduce interior noise on a nearby aircraft fuselage structure in a laboratory. ${ }^{1}$ Control of the shock at the nozzle lip means either (1) the shock is weakened or is converted into an expansion wave, all or in part, or (2) the vibrating lip attachment prevents the characteristics originating in the column of the shear layer from sustaining connection with an out-of-phase surface vibration. Shock from the jet induces high loading by the fundamental and harmonics tones at different locations on the structure. Controlling the shock at the nozzle exit has a greater advantage than controlling the structure.

In the experiment, a model jet is used with a fullscale frame-stringer structure. As a result, the structure responds at a higher frequency compared with a fullscale nozzle placed at an equivalent geometrical distance. The jet flow is supersonic with an underexpanded shock. Active control of the shock

\footnotetext{
"Senior Research Scientist, Associate Fellow
}

at the nozzle exit is achieved by replacing the rigid lip with one oscillating at the shock fundamental frequency. 1,2

Time series of the experimental data are used to unravel the complicated dynamics of the shock motion superimposed on the broadband response using methods described by Abarbanel ${ }^{3}$ Abarbanel, Brown, and Kadthe; ${ }^{4}$ and Maestrello. ${ }^{5}$ The pressure field develops into spirals and flapping nonaxisymmetric modes. The dynamics are captured by arrays of pressure transducers and used in the development of the shock control device. The structural loading due to the shock fundamental and harmonics superimposed on the broadband response exhibits highly complex spatial chaotic deformation patterns of the type described by Dowell, ${ }^{6}$ Nayfeh, ${ }^{7}$ and Dowell. ${ }^{8}$ The loading is sensitive to the initial condition due to the rotating and counterrotating spirals randomly shed from the nozzle lip. Shock shells form between the shear layer and the lip. The tone is produced by the imperfectly expanded jet. The literature refers to this as a screech tone. ${ }^{9}$ Hydrodynamics and structural stability analysis are the recognized tools that provide the loading description.

Early experiments on shock jet noise by Powell ${ }^{9}$ first recognized that the instability of the jet column due to the feedback loop between fluid flow and sound is the cause of a powerful acoustic tone. He proposed a simple mechanism for screech noise; he assumed that the sound waves moving upstream are of sufficient strength to govern the stability of the boundary layer near the exit. Later works by Westley and Woolley; 10 Norum; ${ }^{11}$ Seiner; 12 Ponton and Seiner;13 Tam; ${ }^{14}$ Morris, Bhat, and Chen; ${ }^{15}$ and others indicated that the self-excited oscillation involved the transfer of energy from one wave to another and the spinning of the shock is associated with large-scale instability and sound. Tam presented theoretical arguments to show that the acoustic waves which radiate from the nozzle lip are confined to a relatively narrow frequency band if they are generated by the interaction between the large-scale instability waves and the quasi-periodic shock-cell structure of the jet. 
Section 2 of this paper describes the instrumentation and active controller. The tools used to analyze the dynamics response are described in section 3. Section 4 describes the results with section 4.1 describing the near-field pressure and shock control and section 4.2 describing the response of the structure with and without shock control.

\section{Instrumentation and Active Controller}

The experiment is carried out inside an anechoic chamber. The structure consists of six full-size aluminum aircraft-type panels, 45.72 by 17.71 by $0.1016 \mathrm{~cm}$, with frame and stringers that are mounted on a rigid baffle and placed in the vicinity of the jet exhaust (Fig. 1). The baffle is inclined at an angle of $12^{\circ}$ with the jet axis such that the jet plum will not interact with the baffle. ${ }^{1}$ The structure represents a section of a full-scale aircraft fuselage sidewalk. A convergent nozzle with diameter $D$ of $5.08 \mathrm{~cm}$ is used at a constant pressure ratio $p_{0} / p_{a}$ of 3 and stagnation temperature of $35^{\circ} \mathrm{C}$, with corresponding Reynolds number $U D / N$ of $1.3 \times 10^{6}$ and Strouhal number $f D / U$ of 0.28 , where $p_{0}$ and $p_{a}$ are the stagnation and atmospheric pressure and $f, U$, and $v$ are the shock fundamental frequency, jet exit flow velocity, and kinematic viscosity, respectively. The acoustic pressure radiated from the jet is measured by arrays of unvented, phase-amplitude-matched pressure transducers that are capable of direct current $(\mathrm{dc})$ response and placed in the vicinity of the nozzle exit. The vibration response of the structure is measured by strain gauges located at the midpoint of the bottom panel and side edges of the panel (Fig. 1). This location is the most sensitive one for capturing the passage of the shock during the rotation and flapping cycles.

A new idea for controlling the shock at the exhaust is achieved by replacing the rigid nozzle lip with an oscillating one. The standard round nozzle and two views of the standard nozzle with an oscillating ring placed at the lip of the standard nozzle are shown in Figure 2. The ring is coaxial with the jet and oscillates with the same frequency as the fundamental frequency of the shock. The ring is activated by a feedback loop from the pressure transducer that is placed in the vicinity of the jet exhaust and supplemented by dc bias and a phase filter.1,16,17 The pressure transducers monitor the output waveform in the vicinity of the exhaust. The ring is activated by two electromagnetic actuators. The pressure transducer at $(-a, 0)$ provides the output signal $A(t)=q(-a, 0, t)$. The accelerometer on the ring provides the input signal $I_{i}(t)=m(0,0, t-b)$,
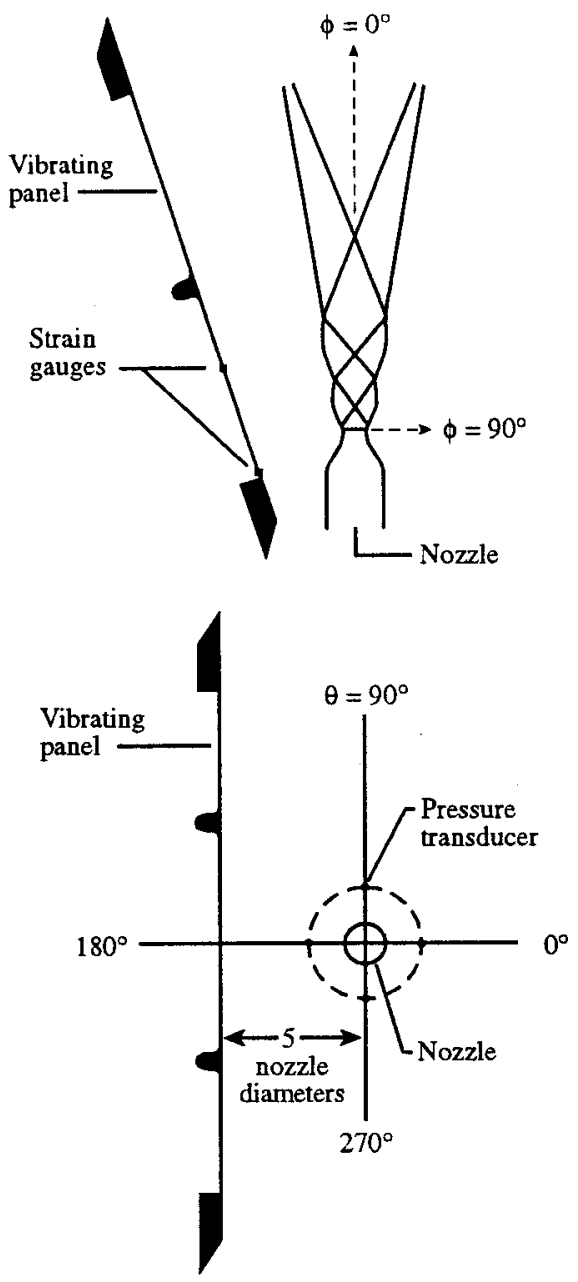

Figure 1. Experimental setting.

where $b$ denotes the time delay or phase shift. The difference $Q(t)$ between input and output is used as a control signal as follows:

$$
C(t)=K(t)\left[I_{i}(t)-A(t)\right]=K(t) Q(t)
$$

where $K(t)$ is an adjustable amplitude. Also we can control the time delay $b(t)$ of $I_{i}(t)$ with respect to $A(t)$. The control is introduced into the system input as a negative feedback $(K>0)$. When the control is accomplished, the output signal $A(t)$ is close to $I_{i}(t)$, and hence the controlling force $C(t)$ becomes small. In the control stage, at least two control level adjustments in $K$ and in the phase lag $b$ are made by the feedback system.

\section{Data Analysis}

The tools used to analyze the dynamics of the response of the jet near-field pressure and panel 


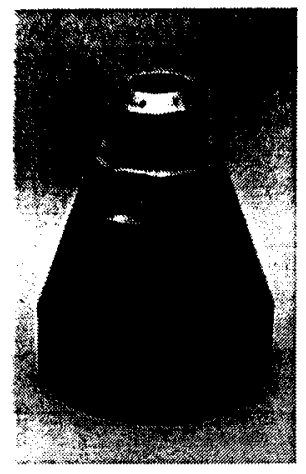

(a) Standard round nozzle.

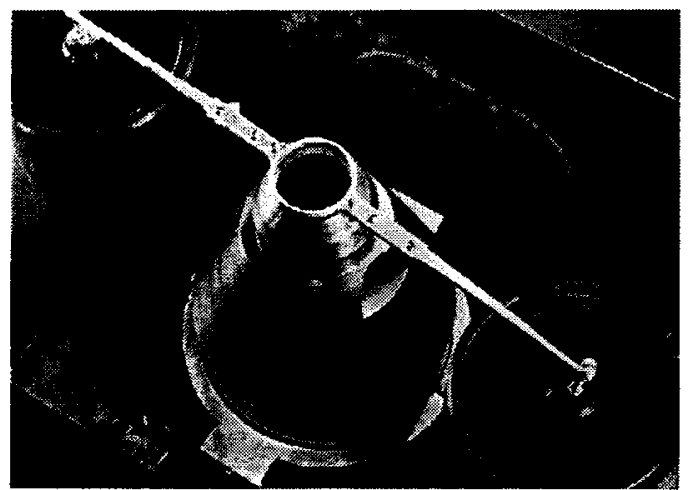

(b) Top view of standard round nozzle with oscillating lip.

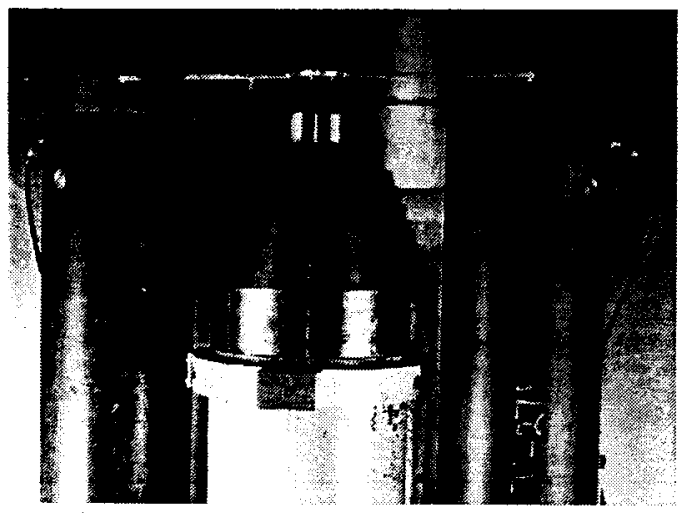

(c) Side view of standard round nozzle with oscillating lip.

Figure 2. Nozzle.

response are explained. ${ }^{1,5}$ Sound from higher speed jets and the resultant structural responses have complex behaviors that are more accuratcly measured by nonlinear system response than by a stochastic system.
Thus the time histories of the near-field pressure from the jet, and the panel strain are obtained. From the time histories, the power spectral density, the phase portrait, and the probability distribution are computed. For a nonstationary signal $q(t, x)$, such as the pressure fluctuation $p(t, x)$ or panel strain $s(t, x)$, the instantaneous power spectrum at instant $T$ is defined by

$$
P(f, T)=\left|\frac{1}{2 \pi} \int_{T-I / 2}^{T+I / 2} \exp (i 2 \pi f t) q(t, x) d t\right|^{2}
$$

where $T$ is chosen so that the experimental run contains the interval $T-I / 2, T+I / 2$ for a sufficiently large $I$. The probability density of $\hat{q}(T, x)$ is denoted by $Q(r, T)$, where

$$
\hat{q}(T, x)=\frac{1}{I} \int_{T-I / 2}^{T+I / 2} q(t, x) d t
$$

and

$$
Q(r, T)=\frac{d}{d r} \operatorname{prob}[\hat{q}(T, x) \leq r]
$$

In chaotic dynamics, searching for a low-dimensional characterization of the system is of great interest. Let $q(t, x)$ be a measured temporal signal or time series at position $x$, which is embedded in a $d$-dimensional phase space by a time delay $\tau$. The set $Z(t)=\left[z_{1}(t), \ldots, z_{d}(t)\right]$ is regarded as a trajectory in the $d$-dimensional phase space. The distance between two points $Z\left(t_{i}\right)$ and $Z\left(t_{j}\right)$ is given by $d_{i j}$, and for a small $\varepsilon>0$, let $N_{d}(n, \varepsilon)$ be the number of pairs of points with distance $d_{i j}<\varepsilon$. Then the correlation sum $C_{d}(\varepsilon)$ and the correlation dimension $D(d)$, for given $d$, are defined by Grassberger and Procaccia ${ }^{18}$ as

$$
C_{d}(\varepsilon)=\lim _{n \rightarrow \infty} \frac{2 N_{d}(n, \varepsilon)}{n(n-1)}
$$

and

$$
D_{d}=\lim _{\varepsilon \rightarrow 0} \frac{\log C_{d}(\varepsilon)}{\log \varepsilon}
$$

For computation, the parameters $\tau$ and $d$ must be chosen properly, and the correlation dimension $D_{d}$ is estimated by

$$
D_{d} \approx \frac{\log C_{d}(n, \varepsilon)}{\log \varepsilon}
$$


for a sufficiently small $\varepsilon$ and large $n$. The estimated dimension $D$ is taken as the asymptotic value of $D_{d}$ as the embedding dimension $d$ increases.

Given the estimated dimension D, the Lyapunov exponent, which is one of the most important characteristics in a dynamic system, can be computed approximately. Several methods exist for computing the Lyapunov exponents. ${ }^{19,18}$ The Eckmann-Ruelle method $^{20}$ is used herein.

Consider $Z(t)$ as the trajectory of a dynamic system in the phase space of dimension $d=D$, obtain the tangent (linear) maps $T_{i}=1,2, \ldots, k$ of this reconstructed dynamical system by a least-squares fit, decompose $T_{i}$ into an orthogonal matrix $Q_{i}$ and an upper triangular matrix by $T_{1}-Q_{1} R_{1}$ and $T_{i} Q_{i-1}=Q_{i} R_{i}$, for $i \geq 2$, and compute the Lyapunov exponents as

$$
\lambda_{i}=\lim \frac{1}{(k-1)} \log \left|\left(R_{k-1} \ldots R_{2} R_{1}\right)_{i i}\right|
$$

for $i=1,2, \ldots, D$. For details see Maestrello ${ }^{5}$ as well as the Eckmann-Ruelle algorithm given by Conte and Dubois. ${ }^{21}$

\section{Results}

The time series of the pressure is used to investigate the dynamics of the convected and radiated pressure from the jet with an oscillating shock. The investigation is carried out in two parts, the first contains the near-field pressure with shock and shock control, and the second contains the corresponding uncontrolled and controlled responses of structure.

\subsection{Near-Field Pressure and Shock Control}

The measured real-time near-field pressure $p(t)$, the computed power spectral density $P(f, T)$, the phase plot of the computed $\dot{p}(t)$ versus $p(t)$ are shown in Figure 3(a), (b), and (c). The real-time pressure near the instant $T$ is used to evaluate the instantaneous plots of spectrum and phase. The shock produced by the underexpanded jet consists of a fundamental and two harmonic tones or screech, $f_{S}, 2 f_{S}$, and $3 f_{S}$. The shock fundamental dominates the broadband spectrum by as much as $40 \mathrm{~dB}$. Figure 4 shows the real-time pressure at $\theta=0^{\circ}, 90^{\circ}, 180^{\circ}$, and $270^{\circ}$ in the same location as Figure 3 over extended time. We see that the envelop of the pressure fluctuation is nearly periodic but the waveform is not. The pressure describes the position of

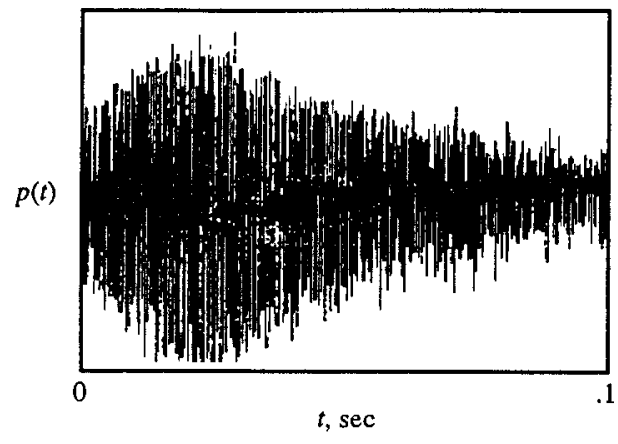

(a) Near-field pressure.

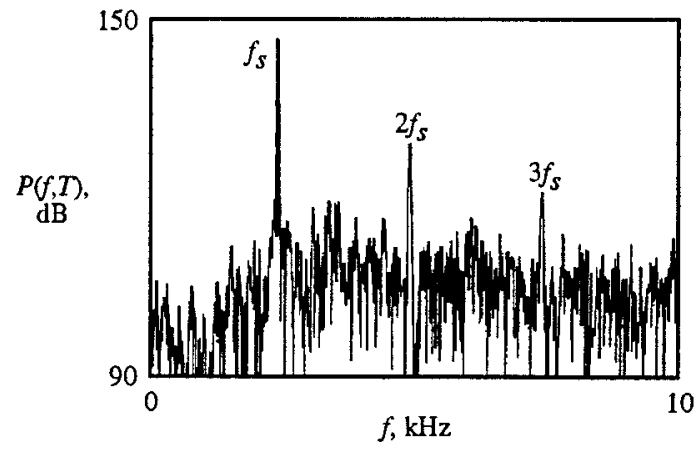

(b) Power spectral density.

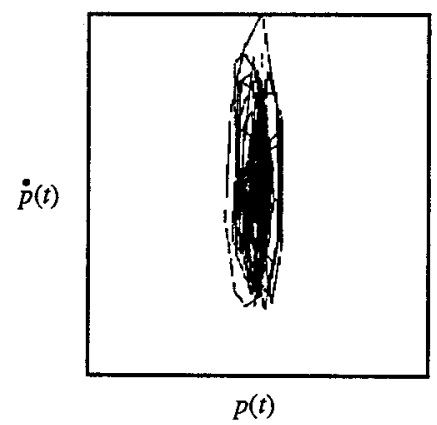

(c) Phase plot.

Figure 3. Pressure about nozzle exit for standard nozzle with $\phi=90^{\circ}$ and $\theta=0^{\circ}$.

the shear layer at any instant and is bounded by an envelope formed by the amplitude of the curve. These rapid changes overshoot the curve of the steady state. The oscillation rises above the starting level; after reaching maximum, the amplitude decreases to the starting value. Azimuthally there is a good correlation between data at two diametrically opposite points. We can trace the occurrence of a peak (or valley) at different angular locations to illustrate the asymmetry of the shock wave from the jet exit. This occurrence suggests a flapping motion of the shear layer. The phase 

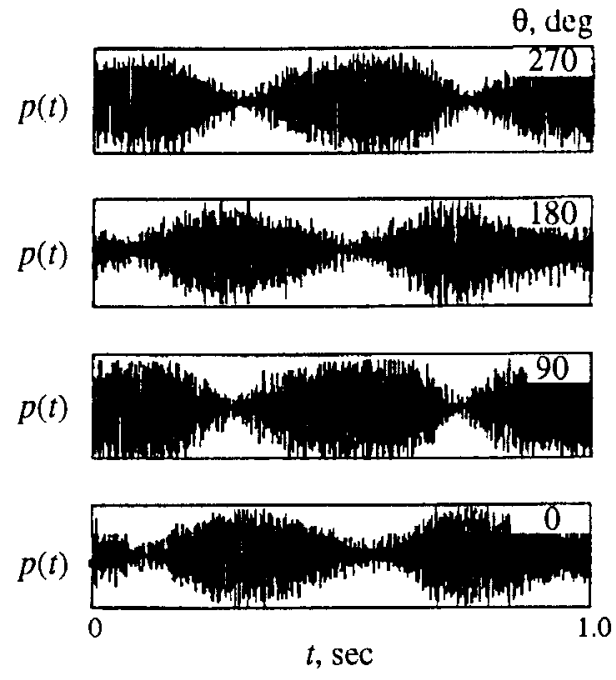

Figure 4. Pressure about nozzle exit for standard nozzle with $\phi=90^{\circ}, \theta=0^{\circ}, 90^{\circ}, 180^{\circ}$, and $280^{\circ}$.

plot is skewed, indicative of rotation in the azimuthal plane. In the meridian plane, $\theta=0^{\circ}$, previous measurements ${ }^{1}$ show the winding and unwinding motion of the jet column going through amplitude and bandwidth changes. These changes are due to the spreading of the shear layer as the velocity varies downstream. The slow drift of the wave modes could be attributed to the convection rotation and counterrotation effects, a result of the helical modulation of the pressure field, discussed also by Ponton and Seiner ${ }^{13}$ and Westley and Woolley. ${ }^{10} \mathrm{Tam}^{14}$ determined the screech frequency by using hydrodynamic stability using multiple scales. This result is in agreement with the experiment.

Control of the shock noise is achieved by replacing a rigid lip with an oscillating one. The new lip consists of a ring coaxial with the jet and oscillating with the same frequency as the fundamental of the attached shock. The control system is similar to the one adapted to control the structure response due to shock impingement. ${ }^{1,2,22}$ The real-time pressure near the nozzle lip is used to activate the feedback control loop (Figs. 3 and 4). The ring is activated by two electromagnetic actuators with amplitude and phase variation $K(t)$ and $b(t)$ so that the energy of the harmonics is shifted back into the fundamental of the shock. Then, the controller reduces the amplitude of the fundamental mode of the shock by increasing the amplitude adjusting to the control signal. Control of the shock is not straightforward, and full control is not always achieved in the initial stage of control. Some foreknowledge of the temporal dynamics is required during the initial stage. Several tries are needed to achieve a systematic feedback control, that is, $K(t)$ and $b(t)$, applicable for all initial stages. Shown in Figure 5 are the measured real time pressure $p(t)$, the computed power spectral density $P(f, T)$, and the phase plot $\dot{p}(t)$ versus $p(t)$ about the nozzle exit when the shock fundamental tone and harmonic tones are fully controlled. Control was applied to the fundamental frequency through a phase- and amplitude-tuning procedure in two stages to control the structural loading. Once control is achieved, the shear layer remains steady from flapping; thus, control is

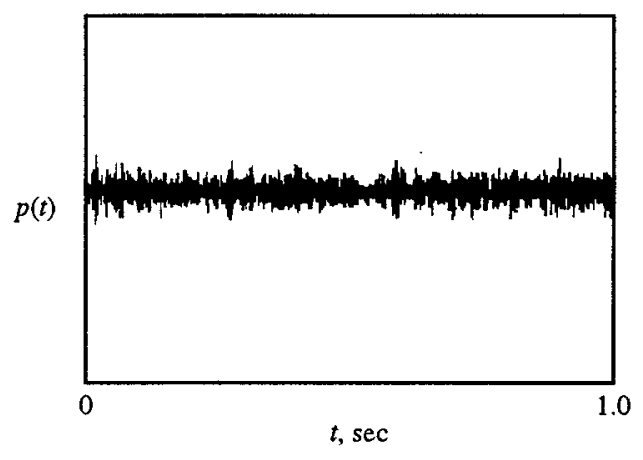

(a) Near-field pressure.

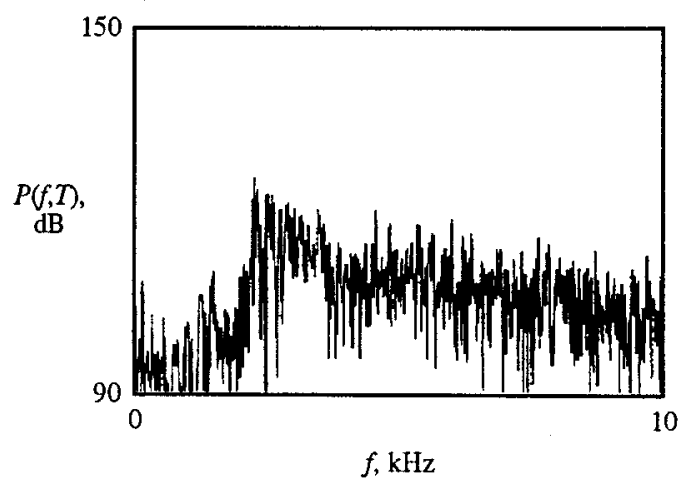

(b) Power spectral density.

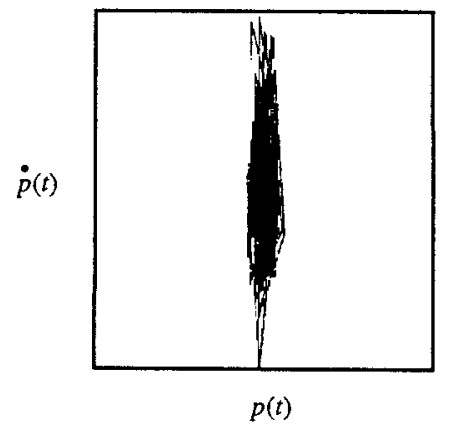

(c) Phase plot.

Figure 5. Pressure about nozzel exit for standard nozzle with oscillating lip with $\phi=90^{\circ}$ and $\theta=0^{\circ}$. 
maintained. When comparing Figure 5 with Figure 3, one notices a remarkable change in near-field pressure. The shock amplitude fundamental and harmonics reduce by several orders of magnitude to the broadband level. Similar changes occur to real-time plot, phase, and probability. Therefore, one can control the shock dynamics from the nozzle lip, rather than controlling the structure loading due to shock impingement.

\subsection{Response of Structure Without and With Shock Control}

The strain response exhibits broadband behavior with sharp, distinct, high-amplitude spikes from the shock impingement by the fundamental and harmonics. The measured real-time strain $s(t)$, the computed power spectral density $S(f, T)$, the phase plot of the computed $\dot{s}(t)$ versus $s(t)$, and the probability $Q(r, T)$ are shown in Figure 6. The shock-induced oscillation dominates the response, the flapping of the shear layer induces random modulation as indicated by the real-time strain, the spectrum indicates the presence of the fundamental and two harmonics, the phase indicates rotation of the shear layer, and the nonsymmetric probability comes from the nonsymmetry in the panel deflection. Thus, results strongly indicate that the response of the structure is the image of the pressure loading.

The flapping of the shear layer maintains some regularity part of the time (Fig. 4); the effect on the panel is not regular as shown by the real-time plot in Figure 6(a). The response of the structure is further analyzed by using the time series of experimental data of the panel response in order to evaluate the correlation dimension $\mathrm{D}$ and Lyapunov exponent $\lambda$ by using the Grassberger and Procaccia algorithm. ${ }^{18}$ The dimension of the attractor is related to the number of degrees of freedom of the panel response, as described by Ruelle and Screenivasan. 19,23 Figure 7 shows the computed exponents, $\lambda_{2}, \lambda_{3}$, and $\lambda_{4}$, versus the embedding time $(D-1) \tau$ from the temporal strain of the panel response with shock loading (Fig. 6), where $D$ is the correlation dimension and $\tau$ is the time delay. The exponents are positive, indicating nonlinear response behaviors. Only the largest Lyapunov exponent is shown for the experimental data. The results give some information about the strain response of the structure which indicates temporal chaotic behaviors when forced by the sound from a supersonic jet with shock.

Once shock is controlled at the nozzle lip, the response of the structure remains temporally smooth over the broadband. Comparing the plot of Figure 8

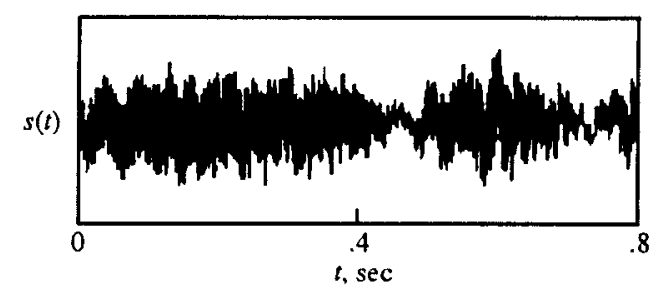

(a) Real-time stain.

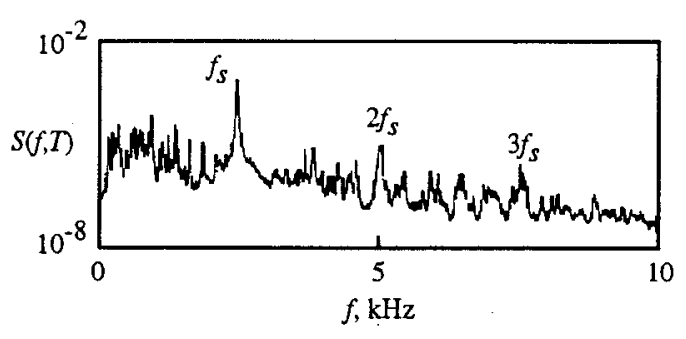

(b) Power spectral density.

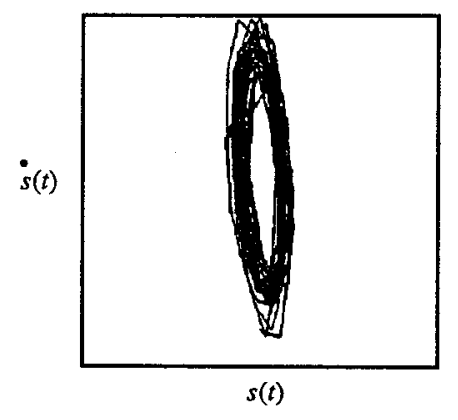

(c) Phase plot.

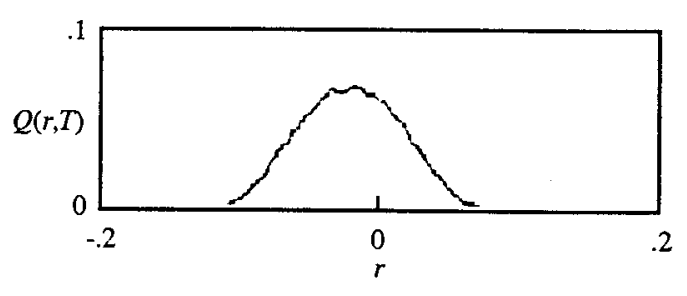

(d) Probability.

Figure 6. Panel strain induced by sound of jet exhausting from standard nozzle.

with that of Figure 6 , we see the remarkable changes in response. The response of the fundamental and harmonics due to shock impingement after control reduces to the equivalent broadband response level. Similar changes are observed in the time plot, phase, and probability. Therefore, controlling the shock at the nozzle lip rather than over the structure is of great advantage, for several reasons, one of which requires a single controller, whereas controlling the structure 


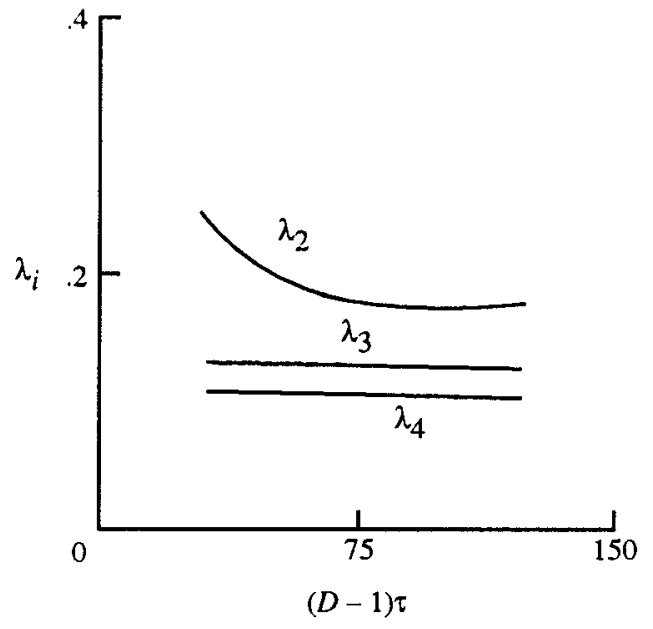

Figure 7. Lyapunov exponent function of embedding time.

requires several controllers because the shock fundamental and harmonics radiate over a wide area and at different angles. 22,17

\section{Conclusions}

A technique is demonstrated for controlling the shock from an underexpanded supersonic jet. A modified nozzle with a vibrating ring becomes an efficient shock suppresser. The shock free jet is a quiet and more efficient jet. The response of the structure also is significantly reduced. The technique is not limited to a circular geometry; other geometries can be used as well. The controller can also be utilized when the nozzle pressure varies.

Two kinds of instability are induced by the shock or screech: a rapid flapping or pulsation of the jet column to each side and helical disturbance along the jet column with winding and unwinding motions. Activating the nozzle lip in a feedback loop prevents the shock wave from sustaining a connection with lip surface; as a result, both the flapping and helical instabilities decay.

The ability to control the shock is of much practical importance. The quality and consistency of the results suggest that a full-scale experiment would be relevant.

\section{References}

1. Maestrello, L., "Control of NonlinearNonstationary Structural Response and Radiation Near a Supersonic Jet," AIAA Journal, Vol. 32, No. 7, July 1994, pp. 1367-1376.

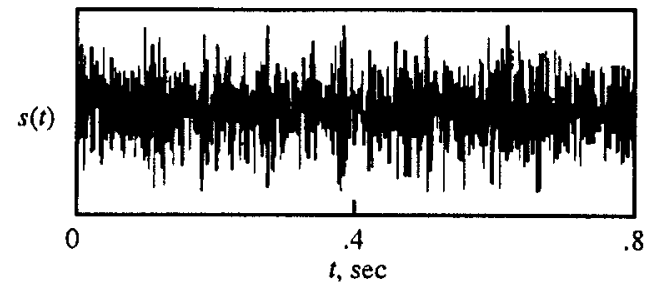

(a) Real-time stain.

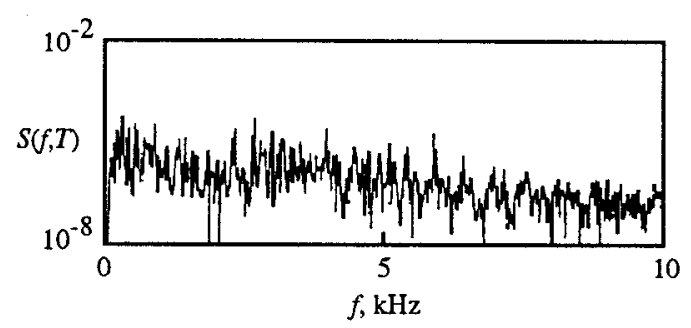

(b) Power spectral density.

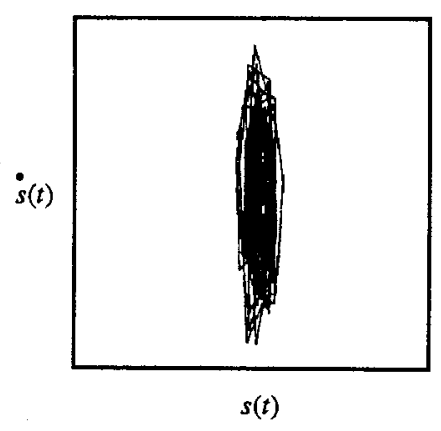

(c) Phase plot.

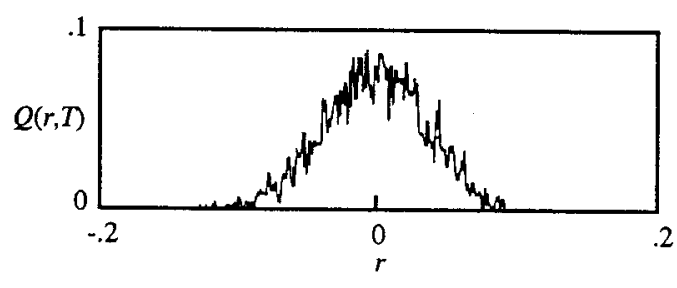

(d) Probability.

Figure 8. Panel strain induced by sound of jet exhausting from standard nozzle with oscillating lip.

2. Maestrello, L., "Control of Sound From HighSpeed Jets Using an Oscillating Lip and a Porous Plug Nozzle," Structural Dynamics: Recent Advances-Proceedings of the 5th International Conference, Vol. 2, edited by N. S. Ferguson, H. F. 
Wolfe, and C. Mei, University of Southampton, UK, 1994, pp. 1045-1055.

3. Abarbanel, H. D. I., "Analysis of Observed Chaotic Data," Springer-Verlag, Berlin, 1996, pp. 25-112.

4. Abarbanel, D. I., Brown, R., and Kadtke, J. B., "Prediction of Chaotic Nonlinear Systems: Methods for Time Series With Broadband Fourier Spectra," Physical Review A: General Physics, Vol. 41, 1990, pp. 1782-1807.

5. Maestrello. L., "Chaotic Response of Panel Vibrations Forced by Turbulent Boundary Layer and Sound," AIAA Journal, Vol. 37, No. 2, February 1999.

6. Dowell, E. H., "Aeroelasticity of Plates and Shells," Noordhoff International Pub., Leyton, The Netherlands, 1975.

7. Nayfeh, A. H., "Nonlinear Propagation of Waves Induced by General Vibration of Plates," Journal of Sound and Vibration, Vol. 79, No. 3, 1981, pp. 429-437.

8. Dowell, E. H., "Chaotic Oscillations in Mechanical Systems," Computational Mechanics, Vol. 3, Springer-Verlag, Berlin, 1988, pp. 199-216.

9. Powell, A., "On the Mechanism of Choked Jet Noise," Proceedings of the Physical Society of London, Vol. PE12, No. 408B, 1953, pp. 10391056.

10. Westley, R., and Woolley, J. H., "The Near Field Sound Pressure of a Choked Jet When Oscillating in the Spinning Mode," AIAA Paper 75-479, March 1975.

11. Norum, T. D., "Screech Suppression in Supersonic Jets," AIAA Journal, Vol. 21, No. 2, February 1983, pp. 235-240.

12. Seiner, J. M., "Advances in High Speed Jet Aeroacoustics," AIAA Paper 84-2275, October 1984.

13. Ponton, M. K., and Seiner, J. M., "Large Scale Structures of Axisymmetric Supersonic Flows,"
Proceeding of the Workshop on Nonstationary Stochastic Processes and Their Applications, World Scientific, Singapore, 1991, pp. 38-47.

14. Tam, C. K. W., "The Shock-Cell Structures and Screech Tone Frequencies of Rectangular and Non-Axisymmetric Supersonic Jets," Journal of Sound and Vibration, Vol. 121, No. 1, 1988, pp. 135-147.

15. Morris, P. J., Bhat, T. R. S., and Chen, G., "A Linear Shock-Cell Model for Jet of Arbitrary Exit Geometry," Journal of Sound and Vibration, Vol. 132, 1989, pp. 199-211.

16. Ott, E., Grebogi, C., and Yorke, J. A., "Controlling Chaos," Physical Review Letters, Vol. 64, No. 1, 1990, pp. 1196-1199.

17. Maestrello, L., "Active Control of Panel Oscillation Induced by Accelerated Turbulent Boundary Layer and Sound," AIAA Journal, Vol. 35, No. 5, May 1997, pp. 796-801.

18. Grassberger, P., and Procaccia, I., "Measuring the Strangeness of Strange Attractors," Physica, Vol. 9D, 1983, pp. 189-208.

19. Ruelle, D., "The Claude Bernard Lecture, 1989, Deterministic Chaos: The Science and Fiction," Proceeding of the Royal Society of London, Vol. A472, 1990, pp. 241-248.

20. Eckmann, J. P., and Ruelle, P., "Ergotic Theory Strange Attractor," Review of Modern Physics, Vol. 5, 1985, pp. 617-656.

21. Conte, R., and Dubois, M., "Lyapunov Exponents of Experimental Systems," Nonlinear Evolutions, edited by J. P. D. Leon, World Scientific, Singapore, 1988, pp. 767-780.

22. Maestrello, L., "Fuselage Structure Response to Boundary Layer, Tonal Sound, and Jet Noise," AIAA 98-2276, June 1998.

23. Screenivasan, K. R., "Transition and Turbulence in Fluid Flow and Low Dimensional Chaos," Frontier in Fluid Mechanics, edited by S. H. Davis and J. L. Lumley, Springer-Verlag, Berlin, 1985, pp. 41-67. 